\title{
Uma análise crítica sobre o uso dos diversos métodos de sedação consciente na odontologia: revisão atualizada da literatura
}

\author{
A critical analysis of the use of different methods of conscious sedation in dentistry: an \\ updated literature review
}
Un análisis crítico del uso de diferentes métodos de sedación consciente en odontología: una revisión bibliográfica actualizada

Carolina Chaves Gama Aires ${ }^{1 *}$, Izabelle Glória dos Santos ${ }^{1}$, Rosa Rayanne Lins de Souza ${ }^{1}$, Aída Juliane Ferreira dos Santos ${ }^{1}$, Igor Chaves Gama da Silva ${ }^{1}$, Maria Eduarda Bené de Oliveira Sabino ${ }^{1}$, Ladyanne Pavão de Menezes ${ }^{2^{\star}}$,Belmiro Cavalcanti do Egito Vasconcelos ${ }^{1}$, Ricardo José de Holanda Vasconcellos ${ }^{1}$.

\section{RESUMO}

Objetivo: Revisar a literatura sobre os diversos aspectos relacionados ao uso da sedação consciente na prática odontológica, bem como os métodos de sedação, visando divulgar o conhecimento sobre essas técnicas. Revisão bibliográfica: $O$ controle da ansiedade é um constante desafio durante 0 atendimento odontológico. A aplicação das técnicas de sedação traz consigo diversas vantagens que auxiliam no tratamento de pacientes especiais e crianças, permitindo a redução dos quadros de ansiedade. Diversas técnicas e fármacos, como os benzodiazepínicos, óxido nitroso, anti-histamínicos, opioides, hidrato de cloral e o fitoterápico Valeriana officinalis foram discutidos no presente estudo, destacando-se os mecanismos de ação, vias de administração, vantagens e desvantagens clínicas e o principais efeitos adversos associados com cada método. Considerações finais: A sedação consciente proporciona um maior conforto durante a realização de tratamentos odontológicos, especialmente em crianças e pacientes com necessidades especiais. Diversos fármacos com propriedades sedativas e hipnóticas estão disponíveis no mercado, cada um com suas vantagens e desvantagens clínicas. O conhecimento sobre as variados métodos capazes de diminuir a ansiedade frente aos tratamentos odontológicos proporciona um melhor controle comportamental e bem estar dos pacientes durantes os atendimentos.

Palavras-chave: Sedação consciente, Ansiolíticos, Óxido Nitroso.

\begin{abstract}
Objective: Review the literature on the various aspects related to use of conscious sedation in dental practice, as well as sedation methods, in order to disseminate knowledge about these techniques. Bibliographic review: Control of anxiety is a constant challenge during dental care. The use of sedation techniques brings with it several advantages that help in treatments of special care patients and children, allowing the reduction of anxiety. Several techniques and drugs, such as benzodiazepines, nitrous oxide, antihistamines, opioids, chloral hydrate and the herbal medicine Valeriana officinalis were discussed in the present study, highlighting the mechanisms of action, administration routes, clinical advantages/disadvantages and the main adverse effects associated with each method. Final considerations: Conscious sedation provides greater comfort during dental treatments, especially in children and patients with special needs. Several drugs with sedative and hypnotic properties are available on the field, each with its clinical advantages and disadvantages. Knowledge about the various methods capable of reducing anxiety provides better behavioral control and comfort of patients during of dental care.
\end{abstract}

Keywords: Conscious Sedation, Anti-anxiety agents, Nitrous oxide.

1 Universidade de Pernambuco (UPE), Recife - PE. *E-mail: carol20101@gmail.com

2 Universidade Federal de Pernambuco (UFPE), Recife - PE. 


\section{RESUMEN}

Objetivo: Revisar la literatura sobre los diversos aspectos relacionados con el uso de la sedación consciente en la práctica odontológica, así como los métodos de sedación, con el fin de difundir el conocimiento sobre estas técnicas. Revisión bibliográfica: El control de la ansiedad es un desafío constante durante la atención odontológica. La aplicación de técnicas de sedación trae consigo varias ventajas que ayudan en el tratamiento de pacientes especiales y niños, permitiendo la reducción de la ansiedad. Varias técnicas y fármacos, como las benzodiazepinas, óxido nitroso, antihistamínicos, opioides, hidrato de cloral y el medicamento a base de hierbas Valeriana officinalis fueron discutidos en el presente estudio, destacando los mecanismos de acción, vías de administración, ventajas y desventajas clínicas y los principales efectos adversos asociados. con cada método. Consideraciones finales: La sedación consciente brinda mayor comodidad durante los tratamientos odontológicos, especialmente en niños y pacientes con necesidades especiales. En el mercado se encuentran disponibles varios fármacos con propiedades sedantes e hipnóticas, cada uno con sus ventajas y desventajas clínicas. El conocimiento sobre los diversos métodos capaces de reducir la ansiedad ante los tratamientos odontológicos proporciona un mejor control del comportamiento y bienestar de los pacientes durante las consultas.

Palabras clave: Sedación consciente, Ansiolíticos, Óxido nitroso.

\section{INTRODUÇÃO}

A ansiedade e o medo são obstáculos durante o tratamento odontológico, tornando a realização do procedimento difícil em pacientes mais ansiosos. O medo e o estresse relacionados com quadros de ansiedade interferem diretamente na procura dos atendimentos odontológicos, gerando um "ciclo vicioso", resultando no aumento da prevalência e agravamento das doenças bucais (FIORILLO L, 2019; TSHISWAKA SK e PINHEIRO SL, 2018).

Essa fobia a tratamentos odontológicos pode ter origem a partir de experiências anteriores desagradáveis, como por exemplo, a anestesia local que é um dos grandes geradores de ansiedade odontológica, bem como os próprios instrumentos e equipamentos odontológicos. Muitas vezes, as experiências negativas dos pais podem influenciar o comportamento dos filhos no consultório odontológico (BATISTA TRM, et al.,2018).

A sedação consciente é uma técnica na qual um ou mais fármacos são empregados para induzir um estado de depressão do sistema nervoso central possibilitando a realização do tratamento (SIVARAMAKRISHNAN G e SRIDHARAN K, 2017). Durante esse estado controlado de depressão consciente medicamentosa, o paciente preserva seus reflexos protetores, tais quais a manutenção do processo respiratório, além de apresentar uma resposta apropriada aos estímulos físicos e comandos verbais (BAEDER FM, et al., 2016).

Este método vem demonstrando ser uma ferramenta eficiente no controle da ansiedade e aversão ao Cirurgião Dentista, muitas vezes se tornando indispensável para a execução de um tratamento (KAPUR A e KAPUR V, 2018). Além de controlar fenômenos de estresse e medo, tem o benefício de ser seguro, mesmo quando utilizado em um ambiente ambulatorial (FIORILLO L, 2019). Para isso, é necessário que os fármacos utilizados tenham uma margem de segurança ampla e satisfatória, tornando improvável a completa perda de consciência (KAPUR A e KAPUR V, 2018).

A aplicação das técnicas de sedação traz consigo diversas vantagens que auxiliam na prática odontológica. Além da redução da ansiedade, também permite a realização do tratamento em pacientes que apresentam intensos reflexos de vômito ou que apresentem necessidades especiais. Ademais, durante esse estado de depressão induzida, há uma diminuição da percepção dos estímulos dolorosos (GUERRERO ORTIZ F, et al., 2020).

Através de uma revista da literatura, o objetivo do presente estudo consistiu em discutir o uso da sedação consciente na prática odontológica, bem como os métodos de sedação, visando divulgar o conhecimento destas técnicas, incrementando o arsenal do cirurgião-dentista no controle da ansiedade e do medo odontológico. 


\section{REVISÃO BIBLIOGRÁFICA}

\section{Níveis de Sedação e indicações da sedação consciente}

A sedação pode ser administrada através de várias rotas como oral, intravenoso, intramuscular, intravenoso e inalatório. De uma forma geral, fatores como o nível de ansiedade, histórico médico do paciente e a complexidade do tratamento determinam a necessidade e o nível de sedação necessários para realização dos tratamentos. São três fatores importantes na avaliação da real necessidade da sedação consciente. Para cada paciente deve ser feito um ajuste individual do nível de sedação para que seja atingido um equilíbrio adequado entre as necessidades do paciente, do operador e para a segurança do procedimento (KAPUR A e KAPUR V, 2018). Os diferentes níveis de sedação, respostas fisiológicas e principais grupos farmacológicos que podem induzir a depressão da consciência podem ser vistos no Quadro 1.

Quadro 1 - Diferentes níveis de sedação.

\begin{tabular}{|c|c|c|c|c|c|}
\hline Modalidade & Respostas & $\begin{array}{l}\text { Respiração e } \\
\text { ventilação } \\
\text { mecânica }\end{array}$ & $\begin{array}{c}\text { Função } \\
\text { cardiovascular }\end{array}$ & $\begin{array}{c}\text { Grupos } \\
\text { farmacológicos }\end{array}$ & $\begin{array}{c}\text { Profissionais } \\
\text { habilitados }\end{array}$ \\
\hline $\begin{array}{c}\text { Sedação } \\
\text { leve } \\
\text { (sedação } \\
\text { mínima ou } \\
\text { ansiólise). }\end{array}$ & $\begin{array}{c}\text { Resposta aos } \\
\text { estímulos verbais. } \\
\text { Função cognitiva e } \\
\text { coordenação física } \\
\text { podem ser } \\
\text { prejudicados } \\
\text { temporariamente. }\end{array}$ & $\begin{array}{c}\text { Sem } \\
\text { alterações. }\end{array}$ & Sem alterações. & $\begin{array}{l}\text { Benzodiazepínicos; } \\
\text { Analgesia inalatória } \\
\text { com } \mathrm{N}_{2} \mathrm{O} / \mathrm{O}_{2} \text { até } \\
20 / 30 \% ; \\
\text { Anti-histamínicos; } \\
\text { Hidrato de cloral; }\end{array}$ & $\begin{array}{l}\text { Médicos; } \\
\text { Cirurgiões- } \\
\text { dentistas. }\end{array}$ \\
\hline $\begin{array}{c}\text { Sedação } \\
\text { Moderada } \\
\text { (sedação } \\
\text { consciente } \\
\text { ou analgesia } \\
\text { relativa). }\end{array}$ & $\begin{array}{l}\text { Resposta aos } \\
\text { estímulos verbais e } \\
\text { estimulação tátil. }\end{array}$ & $\begin{array}{c}\text { Adequada e } \\
\text { nenhuma } \\
\text { intervenção } \\
\text { necessária. }\end{array}$ & $\begin{array}{c}\text { Geralmente } \\
\text { mantida. }\end{array}$ & $\begin{array}{c}\text { Benzodiazepínicos; } \\
\text { Analgesia inalatória } \\
\text { com } \mathrm{N}_{2} \mathrm{O} / \mathrm{O}_{2} \text { até } \\
35 / 50 \% \\
\text { Barbitúricos; } \\
\\
\text { Opioides e propofol } \\
\text { em doses mínimas } \\
\text { (médicos a nível } \\
\text { hospitalar); }\end{array}$ & $\begin{array}{l}\text { Médicos e } \\
\text { Cirurgiões- } \\
\text { dentistas. }\end{array}$ \\
\hline $\begin{array}{l}\text { Sedação } \\
\text { profunda. }\end{array}$ & $\begin{array}{l}\text { Resposta a } \\
\text { estímulo repetitivos } \\
\text { ou dolorosos. }\end{array}$ & $\begin{array}{l}\text { Pode ser } \\
\text { necessária } \\
\text { algum suporte } \\
\text { ventilatório. }\end{array}$ & $\begin{array}{c}\text { Geralmente } \\
\text { mantida. }\end{array}$ & $\begin{array}{c}\text { Benzodiazepínicos; } \\
\text { Analgesia inalatória } \\
\text { com } \mathrm{N}_{2} \mathrm{O} / \mathrm{O}_{2} \text { até } \\
55 / 70 \% ; \\
\text { Barbitúricos; } \\
\text { Opioides e propofol; } \\
\text { Cetamina. }\end{array}$ & Médicos. \\
\hline $\begin{array}{l}\text { Anestesia } \\
\text { Geral. }\end{array}$ & $\begin{array}{l}\text { Perda de } \\
\text { consciência e } \\
\text { ausência de } \\
\text { respostas a } \\
\text { estímulos } \\
\text { dolorosos. }\end{array}$ & $\begin{array}{c}\text { Função } \\
\text { respiratória } \\
\text { comprometida } \\
\text { e necessidade } \\
\text { de suporte } \\
\text { ventilatório. }\end{array}$ & $\begin{array}{c}\text { Pode ser } \\
\text { comprometida. }\end{array}$ & $\begin{array}{c}\text { Agentes } \\
\text { anestésicos; } \\
\text { Barbitúricos; } \\
\text { Agentes } \\
\text { miorrelaxantes; } \\
\text { Cetamina. }\end{array}$ & $\begin{array}{c}\text { Médicos } \\
\text { anestesiologistas. }\end{array}$ \\
\hline
\end{tabular}

Fonte: Aires CCG, et al., 2022; adaptado da classificação de sedação de acordo com a American Society of anesthesiologists (ASA), Resolução do Conselho Federal de Medicina (CFM) 1670/03 e Resolução do Conselho Federal de Odontologia (CFO) 51/2004.

No âmbito da Odontologia, a Resolução Conselho Federal de Odontologia no 51/04, de 30 de abril de 2004 regulamentou normas para habilitação do Cirurgião-Dentista na aplicação da analgesia relativa ou sedação consciente com o intuito de definir os parâmetros legais referentes a essa prática, desde que o cirurgiãodentista esteja devidamente habilitado através de curso autorizado pelo CFO (MINISTÉRIO DA SAÚDE, 2004). 
A sedação consciente está indicada para a ansiedade aguda relacionada à tratamentos odontológicos, procedimentos invasivos e longos, condições médicas agravadas pelo estresse, tais quais angina, asma e epilepsia, pacientes com dificuldades cognitivas e crianças acima de 1 ano de idade (KAPUR A e KAPUR V, 2018). Apesar dos benefícios associados ao emprego dos métodos de sedação, a anestesia geral pode estar melhor indicada em casos de pacientes agressivos ou com muitas complicações sistêmicas, principalmente nos tratamentos mais invasivos (GENTZ R, et al., 2017).

\section{Principais fármacos utilizados na sedação consciente}

\section{Benzodiazepínicos}

Benzodiazepínicos são os principais ansiolíticos utilizados para o manejo da ansiedade leve e moderada, sendo considerados eficazes e seguros para a sedação. Possui propriedades sedativas e ansiolíticas, anticonvulsivantes, capacidade de induzir o relaxamento muscular, além de fornecer a amnésia anterógrada. Contudo, vale salientar, que os benzodiazepínicos não possuem propriedades analgésicas. $\mathrm{Na}$ clínica odontológica, os benzodiazepínicos são os fármacos mais administrados como agentes farmacológicos na sedação consciente por via oral (BAEDER FM, et al., 2016).

O mecanismo de ação se dá através da facilitação da abertura de canais de cloreto mediado pelo ácido gama-aminobutírico (GABA), um neurotransmissor inibitório do Sistema Nervoso Central. A interação do GABA com os receptores benzodiazepínicos provoca a hiperpolarização da membrana neuronal, reduzindo sua excitabilidade, o que altera as habilidades cognitivas do indivíduo. Todos os benzodiazepínicos possuem mecanismos de ação semelhantes, porém com início e duração da ação diferentes (KAPUR A e KAPUR V, 2018).

Os métodos de sedação com os benzodiazepínicos podem ser por via oral ou parenteral. A via oral é a mais usada e possui um início de ação e recuperação mais lenta quando comparada às vias parenterais. $A$ sedação intravenosa tem ação imediata, porém requer habilidade e experiência em administração intravenosa de medicamentos. A via intramuscular é mais lenta que a intravenosa, além de ser inconstante (FIORILLO L, 2019;. GENTZ R, et al., 2017).

Diazepam e midazolam são benzodiazepínicos que têm se mostrado seguros, eficazes e com ampla margem de segurança para sedação consciente. Possuem uma grande margem de segurança clínica, pois a dose tóxica é de 30 a 40 vezes maior do que a dose terapêutica. O midazolam apresenta um início de ação mais rápido que o diazepam, uma meia-vida plasmática mais curta e apresenta uma potência três a quatro vezes maior (KAPUR A e KAPUR V, 2018; CAVALCANTE LB, et al., 2011). O temazepam é outro benzodiazepínico que tem rápido início de ação, meia-vida curta (8 horas) e também é preferível ao diazepam (FIORILLO L, 2019). Na Tabela 1, pode-se observar as dosagens usuais, início e meia vida plasmática dos principais benzodiazepínicos usados, por via oral.

Tabela 1 - Principais benzodiazepínicos usados para induzir a sedação consciente em Odontologia, por via oral.

\begin{tabular}{|c|c|c|c|c|c|c|}
\hline $\begin{array}{c}\text { Nome } \\
\text { genérico }\end{array}$ & $\begin{array}{c}\text { Nome } \\
\text { comercial }\end{array}$ & $\begin{array}{l}\text { Dosagem } \\
\text { em adultos }\end{array}$ & $\begin{array}{l}\text { Dosagem em } \\
\text { crianças }\end{array}$ & $\begin{array}{l}\text { Dosagem } \\
\text { em idosos }\end{array}$ & $\begin{array}{l}\text { Início de } \\
\text { ação } \\
\text { (minutos) }\end{array}$ & $\begin{array}{c}\text { Meia-vida } \\
\text { plasmática } \\
\text { (horas) }\end{array}$ \\
\hline Diazepam & Valium $^{\circledR}$ & 5 a $10 \mathrm{mg}$ & $\begin{array}{c}0,2 \text { a } 0,5 \\
\mathrm{mg} / \mathrm{Kg}\end{array}$ & $5 \mathrm{mg}$ & $45-60$ & $20-50$ \\
\hline Lorazepam & Lorax $^{\circledR}$ & $1 \mathrm{a} 2 \mathrm{mg}$ & $\begin{array}{c}\text { Não } \\
\text { recomendado }\end{array}$ & $1 \mathrm{mg}$ & $60-120$ & $12-20$ \\
\hline Alprazolam & Frontal ${ }^{\circledR}$ & $\begin{array}{c}0,25 \text { a } 0,75 \\
\mathrm{mg}\end{array}$ & $\begin{array}{c}\text { Não } \\
\text { recomendado }\end{array}$ & $0,25 \mathrm{mg}$ & $60-90$ & $12-15$ \\
\hline Midazolam & Dormonid $^{\circledR}$ & 7,5 a $15 \mathrm{mg}$ & 0,3 a $0,5 \mathrm{mg} / \mathrm{kg}$ & $7,5 \mathrm{mg}$ & $30-60$ & $1-3$ \\
\hline Triazolam & Halcion $^{\circledR}$ & $\begin{array}{c}0,125 \text { a } 0,25 \\
m g\end{array}$ & $\begin{array}{c}\text { Não } \\
\text { recomendado }\end{array}$ & $\begin{array}{c}0,06 \mathrm{a} \\
0,125 \mathrm{mg}\end{array}$ & $30-60$ & $1,7-5$ \\
\hline
\end{tabular}

Fonte: Aires CCG, et al., 2022; adaptado de Gallagher C, 2016, Cogo K, et al., 2006 e Cavalcante LB, et al., 2011. 
Os benzodiazepínicos apresentam baixa incidência de efeitos adversos, visto a sua grande margem de segurança clínica, particularmente em tratamentos de curta duração. Ainda assim, alguns pacientes (principalmente crianças e idosos) podem apresentar o chamado "efeito paradoxal", caracterizado por agressividade, agitação, alucinações e irritabilidade, ao invés do esperado efeito ansiolítico, mesmo em baixas doses (GALLAGHER C, 2016).

O uso destes medicamentos é contraindicado em casos de hipersensibilidade aos componentes da fórmula, usuários e dependentes de drogas, portadores de insuficiência respiratória, devido ao efeito depressor dos benzodiazepínicos e portadores de glaucoma. Quando administrados com outros fármacos, tais quais os opióides, os benzodiazepínicos são capazes de diminuir a frequência respiratória, a pressão arterial e a frequência cardíaca. Estas alterações cardiovasculares são raras quando as doses absorvidas são mínimas (RODRIGUES LWM e REBOUÇAS PD, et al., 2015). O uso de técnicas combinando o óxido nitroso com o midazolam em vez do uso individual das drogas, reduz a dose total do midazolam, alcançando um melhor perfil de segurança e com um nível previsível de sedação para procedimentos odontológicos (SIVARAMAKRISHNAN G e SRIDHARAN K, 2017).

Em casos de reações adversas e complicações durante a sedação com benzodiazepínicos é necessário uso de um fármaco reversor dos efeitos de um benzodiazepínico. Nesses casos, o medicamento de escolha deve ser o Flumazenil (SEELHAMMER TG, et al., 2018). Esse fármaco pode ser administrado por via intravenosa ou via intranasal. O Flumazenil traz rápida reversão de todos os benzodiazepínicos, porém é contraindicado para pacientes que usam benzodiazepínicos para tratamento de desordens convulsivas ou em casos de altas doses de antidepressivos tricíclicos (KAPUR A e KAPUR V, 2018).

No Brasil, os dentistas não têm o hábito de empregar a sedação com benzodiazepínicos frequentemente na clínica, devido a diversas questões, entre elas a deficiência em sua formação e treinamento com em relação a este tipo de medicamento (PINHEIRO MLP, et al.,2014).

\section{Óxido Nitroso}

O óxido nitroso, também conhecido como dióxido de nitrogênio $\left(\mathrm{N}_{2} \mathrm{O}\right)$, gás hilariante ou gás do riso, é um gás inodoro e incolor, que possui propriedades ansiolíticas, analgésicas e amnésicas sendo bastante utilizado nas técnicas de sedação ( $\mathrm{CHI} \mathrm{SI}, 2018$ ). O óxido nitroso é um agente ansiolítico eficaz, não irritante, de baixa solubilidade e que, geralmente, não apresenta efeitos significantes sobre o sistema respiratório e cardiovascular. Assim como os benzodiazepínicos, seu efeito também ocorre pela ativação dos receptores GABA. Seu efeito analgésico se dá pela liberação de peptídeos opioides endógenos alterando a sequência metabólica noradrenérgica e modificando o processo nociceptivo espinhal. Por ter uma ação mais a nível de córtex cerebral, o reflexo laríngeo é mantido durante a sedação (FIORILLO L, 2019).

No Brasil, o uso do óxido nitroso foi regulamentado em 2004 pela resolução CFO-051/2004, artigo VI da Lei no 5.081, que regulamenta o exercício da Odontologia. Essa resolução dispõe sobre o emprego da analgesia e hipnose pelo cirurgião-dentista, desde que este esteja devidamente habilitado através de cursos promovidos entidades de Classe, registradas no Conselho Federal de Odontologia (MINISTÉRIO DA SAÚDE, 2004).

A inalação do óxido nitroso baseia-se na mistura entre o $\mathrm{N}_{2} \mathrm{O}$ e o oxigênio $\left(\mathrm{O}_{2}\right)$, administrados em diferentes porcentagens. Após o fluxo inicial de $100 \%$ de oxigênio, o fluxo do óxido nitroso é liberado de forma incremental, sem ultrapassar o limite máximo de $70 \%$ de $\mathrm{N}_{2} \mathrm{O}$ na mistura de $\mathrm{N}_{2} \mathrm{O}_{2} \mathrm{O}_{2}$. O percentual de $\mathrm{N}_{2} \mathrm{O}$ na mistura deve ser ajustado de acordo a necessidade de cada paciente (LADEWIG VM, et al., 2016). O N $\mathrm{N}_{2} \mathrm{O}$ tem baixa solubilidade tecidual e alta concentração alveolar mínima, o que proporciona um rápido início de ação e uma recuperação rápida, garantindo uma sedação controlada e retorno rápido às atividades cotidianas (KAPUR A e KAPUR V, 2018).

A combinação entre a mistura de $\mathrm{N}_{2} \mathrm{O} / \mathrm{O}_{2}$ e o midazolam, além de ter um melhor efeito quando comparada ao uso individual das drogas, diminui o risco de toxicidade relacionada ao uso do midazolam e melhora a aceitação da máscara nasal em crianças (BLUMER S, et al., 2018; SIVARAMAKRISHNAN G e SRIDHARAN K, 2017). 
Os efeitos adversos mais comuns associados a esta técnica são as náuseas e os vômitos. Outros eventos foram reportados na literatura, tais quais, dor torácica, dessaturação e convulsões tônico-clônicas. Na maioria das vezes, o aparecimento dos efeitos adversos está relacionado com a administração do $\mathrm{N}_{2} \mathrm{O} / \mathrm{O}_{2}$ em concentração mais elevada (>50\%) ou com longos períodos de sedação. Sabendo que a reação às drogas pode variar muito entre os pacientes, com alguns atingindo a concentração alveolar mínima mais rapidamente, a monitorização constante é de extrema importência para evitar uma sedação profunda e garantir a manutenção dos reflexos laríngeos, e consequentemente, a respiração do paciente (CHI SI, 2018).

A sedação com óxido nitroso está contraindicada em pacientes psicóticos, pacientes com obstrução das vias aéreas superiores (inclusive em pacientes acometidos com viroses respiratórias), com doenças sistêmicas graves, ou com problemas pulmonares crônicos, como a doença pulmonar obstrutiva crônica (FIORILLO L, 2019; KAPUR A e KAPUR V, 2018). As principais desvantagens da sedação com óxido nitroso são os custos dos equipamentos necessários, necessidade de treinamento e habilitação do cirurgião-dentista e de sua equipe, infraestrutura e a variação individual (e subjetiva) da dosagem para cada paciente (FIORILLO L, 2019).

\section{Anti-histamínicos}

Os anti-histamínicos são uma classe popular de fármacos com propriedades sedativas, geralmente usado em combinação com outros, como opióides e óxido nitroso, com os efeitos desejados de sedação e hipnose, com a vantagem de não causar depressão cárdio-respiratória ou inconsciência (GENTZ R, et al., 2017). São drogas facilmente encontradas, com um bom custo benefício, seguras do ponto de vista clínico e que podem ser administradas por via oral. O mecanismo de ação desses fármacos deve-se as propriedades anticolinérgicas induzidas pelo bloqueio pós-sinápticos nos receptores dopaminérgicos (MOZAFAR S, et al., 2018).

Hidroxizina e prometazina são os anti-histamínicos mais empregados na sedação consciente, principalmente na odontopediatria. Quando usados em combinação com outras drogas, o efeito antiemético desses anti-histamínicos ajuda a mitigar as náuseas e vômitos induzidos por outros medicamentos (GENTZ R, et al., 2017).

Os efeitos adversos mais comum dos anti-histamínicos são os sintomas extrapiramidais leves, como a inquietação motora, porém esses episódios são raros quando utiliza-se a via oral. Outros efeitos adversos são raros, tais quais a xerostomia, retenção urinária, palpitações, hipotensão, cefaléia, alteração do apetite, constipação ou diarréias (MELONARDINO AP, et al., 2016)

Estudo comparando a prometazina associada ao óxido nitroso versus o midazolam combinado com o óxido nitroso não mostrou diferenças estatisticamente significativas entre os dois protocolos sedativos utilizados na pesquisa, porém a sedação ocorreu mais rapidamente no grupo do midazolam/ $\mathrm{N}_{2} \mathrm{O}$ (MOZAFAR $\mathrm{S}$, et al., 2018).

\section{Opioides}

Os opioides são drogas de efeito analgésicos que se ligam a receptores específicos localizados em todo o sistema nervoso central e outros tecidos. Quando combinados com outros agentes sedativos, essa família de fármacos pode ser uma excelente escolha para melhorar a qualidade da sedação, visto seu grande potencial analgésico. A administração oral é a via preferida, especialmente na odontopediatria, porém a sua absorção não é confiável resultando em uma eficácia pouco previsível (GENTZ R, et al., 2017).

O fentanil é um opioide de curta duração (30 a 60 minutos), 60 a 80 vezes mais potente do que a morfina e de rápido início de ação. Administrado por via parenteral, transdérmica, nasal ou oral, o fentanil é um fármaco lipofílico que é absorvido na mucosa bucal, metabolizado no fígado, e excretado na urina. Dose recomendada é de $1 \mu \mathrm{g} / \mathrm{kg} /$ dose intravenosa, que pode ser repetido por incrementos de $1 \mu \mathrm{g} / \mathrm{kg}$, se necessário (KAPUR A e KAPUR V, 2018). O uso do fentanil favorece uma sedação mais profunda e pode ocasionar uma depressão respiratória, que pode ser agravada sinergicamente por combinações com medicamentos sedativos, especialmente em pacientes idosos. Em virtude disso, o uso dessa droga deve ser realizada de preferência em ambiente hospitalar com acompanhamento de um médico anestesista (GÖKTAY O, et al., 2011). 
O tramadol é um agente analgésico opioide sintético de ação central, com um efeito analgésico que é mediado principalmente pela inibição da recaptação de norepinefrina e serotonina, além de possuir uma baixa afinidade para receptores opioides, proporcionando alívio de dores moderadas a grave. Ao contrário dos analgésicos opióides tradicionais, como morfina e fentanil, o tramadol preserva as funções respiratórias e cardiovasculares (GÖKTAY O, et al., 2011). O uso do tramadol é amplamente estudado na Odontologia, principalmente para o controle de dores pós-operatórias ou como analgésico preemptivo (ARAÚJO FAC, et al., 2012). Em estudo comparando o uso do fentanil ou do tramadol associados ao midazolam, observou-se que o tramadol também pode ser utilizado com o propósito de melhorar o efeito da sedação consciente, assim como o fentanil, apesar das distintas propriedades farmacocinéticas e farmacodinâmicas entre as drogas (CHON J e LEE J, 2011).

De uma forma geral, os opioides podem causar depressão respiratória e cardiovascular, tontura, xerostomia, hipoventilação, e hipotensão, além de náuseas e vômitos. Os efeitos adversos são geralmente relacionados com a severidade da dor, via de administração, dosagem e combinação com outras drogas. Os efeitos adversos dos opioides podem ser revertidos com o uso da naloxona (KAPUR A e KAPUR V, 2018; GENTZ R, et al., 2017).

\section{Hidrato de cloral}

O hidrato de cloral foi um dos agentes hipnótico e sedativo mais utilizados na sedação consciente, especialmente em crianças. É um fármaco derivado do álcool, que é rapidamente absorvido pelo trato gastrointestinal após a administração oral. Sofre o metabolismo de primeira passagem no fígado e nos rins, onde é convertido em sua forma ativa, o tricloroetanol. Por fim é metabolizado no fígado e excretado na urina (SONG S, et al., 2020).

Tem um início de ação em cerca de 30 a 60 minuto e dose recomendada de 10 a $50 \mathrm{mg} / \mathrm{Kg}$ (CAVALCANTE LB, et al., 2011). No entanto, mesmo em doses terapêuticas, o hidrato de cloral pode enfraquecer o tônus muscular da língua, fazendo com que a língua retraia em direção à parede posterior da orofaringe, obstruindo as vias aéreas. Dessa forma, faz-se necessário o monitoramento adequado do paciente durante todo o procedimento (SONG S, et al., 2020).

Por muito tempo, o hidrato de cloral foi considerado seguro e eficaz com benefícios notáveis, tais quais a fácil administração via oral ou retal e o custo relativamente baixo. Entretanto, o hidrato de cloral apresenta efeitos sedativos inconsistentes, longa duração de ação e propriedades farmacocinéticas menos previsíveis que outros agentes ansiolíticos, como os benzodiazepínicos. Essa droga está associada a vários efeitos adversos, como vômitos, naúseas, hiperatividade (efeito paradoxal), hipoventilação, apnéia, depressão respiratória e e alterações hemodinâmicas. Por essas razões, a produção comercial da solução oral de hidrato de cloral cessou em 2012 nos Estados Unidos. No entanto, as farmácias continuam a preparar outras formulações desse fármaco. $O$ aumento da popularidade dos outros agentes sedativos somados a todos esses fatores podem justificar o desuso do hidrato de cloral na atualidade (FARR KM, et al., 2020).

\section{Valeriana Officinallis}

Atualmente, medicamentos fitoterápicos com propriedades ansiolíticas e hipnóticas têm atraído cada vez mais interesse em diferentes áreas da medicina devido à sua baixa incidência de efeitos colaterais. A Valeriana Officinalis é uma planta herbácea da família Valerianaceae, que tem sido usada como sedativo, antisséptico, anticonvulsivante e analgésico. Estudos recentes demonstraram que a valeriana é clinicamente segura e eficaz no tratamento ansiedade e insônia induzida por estresse, com pouca ou nenhuma efeitos colaterais (FARAH GJ, et al., 2019). Misturas farmacêuticas contendo valeriana extrato têm sido usado em vários países devido à ausência de efeitos colaterais comumente associados aos benzodiazepínicos (PINHEIRO MLP, et al.,2014).

Ao contrário dos benzodiazepínicos, extratos de valeriana não interferem com a coordenação voluntária dos movimentos e fala, não causam danos físicos e/ou dependência psicológica. Ensaios clínicos utilizando a valeriana como agente sedativo em cirurgia de terceiros molares reiteraram o efeito ansiolítico desse fitoterápico. Portanto, a valeriana pode representar uma alternativa viável na sedação moderada, especialmente nos casos em que o uso dos benzodiazepínicos são contraindicados (FARAH GJ, et al., 2019; PINHEIRO MLP, et al., 2014). 


\section{Monitorização durante após o emprego da sedação consciente}

O tratamento odontológico é realizado sob condições vulneráveis à obstrução das vias aéreas, como posição anormal da cabeça, posições da língua e presença de corpos estranhos, incluindo dique de borracha, água exógena, saliva e sangue. Quando esses fatores são associados com níveis excessivos de sedação,medidas especiais devem ser tomadas. Na maioria das vezes, os eventos adversos são fortemente correlacionados com instalações não hospitalares e consultórios dentários (SONG S, et al., 2020).

Dessa forma, todos os profissionais devem estar familiarizados e preparados para emergências, tais quais a obstrução de vias aéreas, anafilaxia, toxicidade das medicações, depressão respiratória e apnéia, aspiração e emergências cardiovasculares agudas. A equipe deve estar atualizada sobre os protocolos de segurança, diretrizes especiais e recomendações que devem ser seguidas, visando a segurança e otimizando a prática odontológica (SAXEN MA, et al., 2019).

A combinação de uma avaliação visual visual (cianose de lábios e mucosas), o uso de oxímetro de pulso, e se disponível, a capnografia aumentam a probabilidade de detectar eventos adversos, possivelmente, reduzindo a necessidade de intervenções nas vias aéreas. O oxigênio suplementar, antes e durante o período da sedação, pode fornecer alguns minutos valiosos em casos de dessaturação, apnéia, depressão respiratória ou parada cardiovascular (SAXEN MA, et al., 2019).

O paciente deve ser monitorado no pós-operatório, em ambiente equipado com um dispositivo de sucção e ventilação por pressão (bolsa-válvula-máscara adequados para a idade e o tamanho do corpo) que possa fornecer oxigênio a 90\%. O paciente só deve ser liberado apresentando funções respiratórias e cardiovasculares estáveis, verbalizando e com funções cognitivas semelhantes ao seu estado prévio a sedação (SONG S, et al., 2020).

\section{CONSIDERAÇÕES FINAIS}

A sedação consciente proporciona um maior conforto durante a realização de tratamentos odontológicos, especialmente em crianças e pacientes com necessidades especiais. Diversos fármacos com propriedades sedativas e hipnóticas estão disponíveis no mercado, cada um com suas vantagens e desvantagens clínicas. Estudos sobre a sedação consciente podem difundir cada vez mais o emprego das diferentes técnicas por um maior número de profissionais. Logo, o conhecimento sobre os variados métodos capazes de diminuir a ansiedade frente aos tratamentos odontotológicos é de extrema importância para minimizar o medo associado aos procedimentos odontológicos, melhorando o controle comportamental e o bem-estar dos pacientes.

\section{REFERÊNCIAS}

1. AMERICAN SOCIETY OF ANESTHESIOLOGISTS (ASA). Practice guidelines for preoperative fasting and the use of pharmacologic agents to reduce the risk of pulmonary aspiration: An updated report. Anesthesiology, 2011; 114: 495511.

2. ARAÚJO FAC, et al. Comparative analysis of preemptive analgesic effect of tramadol chlorhydrate and nimesulide following third molar surgery. Journal of Craniomaxillofacial Surgery. 2012; 40(8): e346-9.

3. BAEDER FM, et al. Conhecimento de pacientes sobre o uso de benzodiazepínicos no controle da ansiedade em Odontologia. Revista da Associação Paulista de Cirurgiões-dentistas. 2016; 70: 1-5.

4. BATISTA TRM, et al. Medo e ansiedade no tratamento odontológico: um panorama atual sobre aversão na odontologia. Salusvita, 2018; 37(2): 449-469.

5. BLUMER S, et al. Oxygen Saturation and Pulse Rate Change in Children during Sedation with Oral Midazolam and Nitrous Oxide. The Journal of Clinical Pediatric Dentistry, 2018; 42: 1-4.

6. CAVALCANTE LB, et al. Sedação consciente: um recurso coadjuvante. Arquivos em Odontologia, 2011; 47(1): 45-50.

7. $\mathrm{CHI}$ SI. Complications caused by nitrous oxide in dental sedation. Journal of Dental Anesthesia and Pain Medicine, 2018; 18(2): 71-78.

8. CHON J, LEE J. Is tramadol better than fentanyl for conscious sedation Journal of Oral Maxillofacial Surgery. 2011; 69(10): 2485- 6.

9. COGO K, et al. Sedação consciente com benzodiazepínicos em odontologia. Revista de Odontologia da Universidade Cidade de São Paulo, 2006; 18(2): 181-8. 
10. FARAH GJ, et al. Assessment of Valeriana officinalis I. (Valerian) for Conscious Sedation of Patients During the Extraction of Impacted Mandibular Third Molars: A Randomized, Split-Mouth, Double-Blind, Crossover Study. Journal of Oral and Maxillofacial Surgery. 2019; 77(9): 1796.e1-1796.e8.

11. FARR KM, et al. Chloral Hydrate Sedation in a Dexmedetomidine Era. Hospital Pharmacy. 2020; 55(4): $236-239$.

12. FIORILLO L. Conscious Sedation in Dentistry. Medicina (Kaunas). 2019;55(12):778.

13. GALLAGHER C. Benzodiazepines: Sedation and Agitation. Dental Update, 2016; 43(1): 83-9.

14. GENTZ R, et al. Safety and Efficacy of 3 Pediatric Midazolam Moderate Sedation Regimens. Anesthesia Progress, 2017; 64(2): 66-72.

15. GÖKTAY O, et al. A comparison of the effects of midazolam/fentanyl and midazolam/tramadol for conscious intravenous sedation during third molar extraction. Journal of Oral Maxillofacial Surgery. 2011; 69(6): 1594-9.

16. GUERRERO ORTIZ F, et al. Sedación consciente, inhalatoria y farmacológica, su efectividad en la reconducción de la conducta del paciente pediátrico en la consulta dental: estudio observacional de corte transversal / Conscious inhalation and pharmacological sedation, its effectiveness in the reconduction of the behavior of the pediatric patient in the dental office: cross-sectional observational study. Avances en odontoestomatología. 2020; 36(4): 180-185.

17. KAPUR A, KAPUR V. Conscious sedation in dentistry. Annals of Maxillofacial Surgery. 2018; 8:320-3.

18. LADEWIG VM, et al. Sedação consciente com óxido nitroso na clínica odontopediatríca. Odontologia Clínica e Cientifica, 2016; 15(2): $91-96$.

19. MELONARDINO AP, et al. Ansiedade: Detecção e conduta em odontologia. Revista Uningá. 2016; 48: 1-8.

20. MINISTÉRIO DA SAÚDE. Resolução CFM nำ1670/2003, de 11 de julho de 2003. Estabelece normas para a prática de sedação consciente ou níveis mais profundos de sedação. 2003. Disponível em: https://saes.org.br/images/meta/1c5c1315-ce4b-4d57-b61c-1becc30acc3b/118/resoluc-a-o-cfm-n-1670-2003-de-11de-julho-de-2003-estabelec.pdf. Acessado em: 28 de setembro de 2021.

21. MINISTÉRIO DA SAÚDE. Resolução Conselho Federal de Odontologia nำ51/04, de 30 de abril de 2004. Estabelece normas para habilitação do Cirurgião-Dentista na aplicação da sedação consciente. 2004. Disponível em: https://www.normasbrasil.com.br/norma/resolucao-51-2004_100558.html. Acessado em: 28 de setembro de 2021.

22. MOZAFAR S, et al. Comparison of nitrous oxide/midazolam and nitrous oxide/promethazine for pediatric dental sedation: A randomized, cross-over, clinical trial. Dental Research Journal. 2018; 15(6): 411-419.

23. PINHEIRO MLP, et al. Valeriana officinalis $L$. for conscious sedation of patients submitted to impacted lower third molar surgery: A randomized, double-blind, placebo-controlled split-mouth study. Journal of Pharmacy \& Bioallied Science. $2014 ; 6(2):$ 109-14.

24. RODRIGUES LWM, REBOUÇAS PD. O uso de Benzodiazepínicos e N2O/O2 na sedação consciente em Odontopediatria. Revista da Faculdade de Odontologia de Lins, 2015; 25: 1-5.

25. SAXEN MA, et al. Mason KP. Advancing the Safe Delivery of Office-Based Dental Anesthesia and Sedation: A Comprehensive and Critical Compendium. Anesthesiology Clinics. 2019; 37(2): 333-348.

26. SEELHAMMER TG, et al. O uso de flumazenil para depressão respiratória associada ao benzodiazepínico na recuperação pós-anestésica: riscos e resultados. Revista Brasileira de Anestesiologia, 2018; 68: 1-7.

27. SIVARAMAKRISHNAN G, SRIDHARAN K. Nitrous Oxide and Midazolam Sedation: A Systematic Review and MetaAnalysis. American Dental Society of Anesthesiology. 2017; 64: 1-7.

28. SONG S, et al. Safety of chloral hydrate sedation in dental practice for children: an overview. Journal of Dental Anesthesia and Pain Medicine. 2020; 20(3): 107-118.

29. TSHISWAKA SK, PINHEIRO SL. Efeito da música na redução da ansiedade em crianças durante o tratamento dentário. Rev. Gaúcha. Odontol. 2018; 68:1 - 8. 